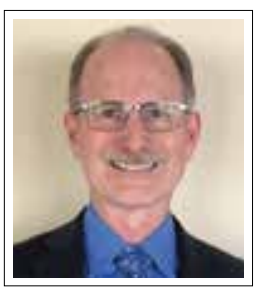

John Hickner, MD, MSc

Editor-in-Chief

doi: $10.12788 /$ fp. 0282

\title{
Let's talk about healthy aging (but where to begin?)
}

$\mathrm{T}$ his month's cover story, "A 4-pronged approach to foster healthy aging in older adults," by Wilson and colleagues (page 376) provides a wealth of information about aspects of healthy aging that we should consider when we see our older patients. After reading this manuscript, I was a bit overwhelmed by the amount of information presented and, more so, by the thought of attempting to incorporate into my practice all of the possible screenings and interventions available to help older adults improve and maintain their health.

There is no debate about the importance of issues such as diet, exercise and mobility, mental health and cognition, vision and hearing, and strong social connections for maintaining health as we age. The difficulty comes in deciding how to spend our limited time with older patients during office encounters. Most older adults have several chronic diseases that need our attention, and they often have various medications that need to be monitored for effectiveness and safety, which can be time consuming. And, of course, we need to take time to screen for cardiovascular risk and cancer, too. Where to start?

Dr. Wilson's solution makes sense to me: Take advantage of the annual wellness visit to discuss diet, exercise, mental health, vision and hearing, and social relationships. I am not so sure, however, if using formal screening instruments is the best way to do this, especially since there is no strong research that demonstrates improved patient-relevant outcomes using screening instruments, except, perhaps, for periodically screening for anxiety and depression.

It may be as effective to use what I will call the "chat technique." Start with open-ended questions and statements, such as: "How are things going for you?" "Tell me about your family." "What do you do for physical activity?" and "How has your mood been lately?"

An excellent complement to the chat technique is the goal-setting approach championed by geriatrician and family physician Jim Mold. ${ }^{1}$ His premise is that health itself is not the most important goal for most people, but rather a means to an end. That end is very specific to every person. An elderly, frail woman's main life goal, for example, might be to remain in her own home for as long as possible or to live long enough to attend her great-grandson's wedding.

Goal setting provides an excellent context for true shared decision-making. I agree with Dr. Wilson's closing statement:

"As family physicians, it is important to capitalize on longitudinal relationships with patients and begin educating younger patients using this multidimensional framework to promote living 'a productive and meaningful life' at any age."

JFP

Reference

1. Mold, JW. Goal-Oriented Medical Care: Helping Patients Achieve Their

Personal Health Goals. Full Court Press; 2017.

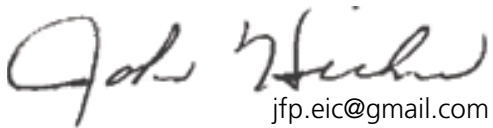

